\title{
Acupuncture for Lateral Epicondylitis
}

Fatma G. UĞURLU1', Salih MOLLAHALILOĞLU², Hüseyin NAZLIKUL

${ }^{1}$ Department of Physical Medicine and Rehabilitation, Faculty of Medicine, Yıldırım Beyazıt University, Ankara, Turkey.

${ }^{2}$ Department of Public Health, Faculty of Medicine, Yıldırım Beyazıt University, Ankara, Turkey.

${ }^{3}$ Department of Physical and Complementary Medicine, Faculty of Medicine, University of Hamburg, Germany.

\section{ABSTRACT}

Lateral epicondylitis (LE), is one of the most common diseases of the arm with repetitive microtrauma by overuse of the wrist. Several conventional therapies are offered for tennis elbow. When classical conservative treatments are unsuccessful or have side effects, patients opt for complementary medicine, particularly needle acupuncture. A 26-year-old male right-handed amateur volleyball player presented with severe pain radiating from the lateral aspect of the right elbow. Pain was initiated by resisted wrist extension with the elbow in full extension and the forearm in pronation. His pain was unresponsive to NSAID, local corticosteroid injection, and 21 times rehabilitation. The body points were selected according to the pulse test in Chinese medicine, and the ear points were determined by detecting the Nogiers' ear acupuncture points. The energy level of the patient was measured using the pulse oscillography. Blocking was observed in all three energy circles on the pulse oscillography. At the end of the therapy, the average grip strength of the affected hand increased and VAS had decreased by the end of the treatment. The pulse oscillography indicated that the blocking and energy imbalance in the three circles were completely dissolved.

In conclusion, acupuncture is an effective therapy for $L E$, and pulse oscillography is an important device for the diagnosis and therapy.

Key words: Lateral epicondylitis, acupuncture

\section{INTRODUCTION}

Lateral epicondylitis (LE), known as tennis elbow, is one of the most common diseases of elbow (1). Its cardinal symptom is tenderness and pain while using the extensor muscles of wrist repetitively. Men and women seem to be equally predisposed to this disease, and the age of onset is usually between 35 and 55 years (2). The incidence is estimated between $1 \%$ and $3 \%$ per year in the adult general population. It occurs because of overuse injury. But a sudden extreme effort or activity such as trauma can also cause damage to the epicondylar area (3). The classical description is pain at the lateral aspect of the elbow that often radiates down the forearm. It leads to the failure of common extensor tendon, specifically the extensor carpi radialis brevis portion. The diagnosis of $L E$ includes palpation of the lateral epicondyle, resisted wrist extension, resisted wrist and middle finger extension, and passive wrist flexion $(4,5)$.

Several conventional therapies are offered for tennis elbow, including non-steroidal anti-inflammatory drugs (NSAID), restricted movement, transcutaneous electrical nerve stimulation, conventional physiotherapy (such as stretching and strengthening exercise), extracorporeal shock wave therapy, low-level laser therapy, focal corticosteriod injection, botulinum toxin injection, gypsum cast, and surgery. Because the process of the disease differs from patient to patient,

Correspondence:

Fatma G. Uğurlu

Department of Physical Medicine and Rehabilitation, Faculty of Medicine, Yıldırım Beyazıt University, Ankara, Turkey.

e-mail: glcnrl@hotmail.com 
choosing an appropriate treatment is difficult. When classical conservative treatments are unsuccessful or have side effects, patients opt for complementary medicine, particularly needle acupuncture (6-8).

In this study, a case of LE treated with needle acupuncture therapy is presented.

\section{CASE REPORT}

A 26-year-old male right-handed amateur volleyball player presented with severe pain radiating from the lateral aspect of the right elbow to the right wrist and limitation of elbow movement for 6 months. He was healthy before this pain. His symptoms began after a fall while playing volleyball. His pain stayed throughout the day and did not resolve with rest. His grip was weak, and he had difficulty in carrying items with his right hand. Because of the pain, he was depressed. He had no rheumatic disease or previous elbow surgery. His lateral epicondyle, slightly distal to the extensor mass, was tender. Pain was initiated by resisted wrist extension with the elbow in full extension and the forearm in pronation. Moreover, his cervical spine and shoulder examination did not reveal abnormal findings. He had no peripheral arthritis. His neurological and systemic examination showed no abnormality, except for the aforementioned findings.

Radiograph, ultrasound, and magnetic resonance imaging were reported as normal. Inflammatory markers, C-reactive protein, erythrocyte sedimentation rate, complete blood count, and hormonal and biochemical markers were in normal ranges. When the patient attended the physical therapy and rehabilitation clinic, he had already been diagnosed with LE, and medical treatment had just been started. His pain was unresponsive to NSAID, local corticosteroid injection, and 21 times rehabilitation.

In the past 10 years, acupuncture has gained wider acceptance for pain. In this case, the body points were selected according to the pulse test in Chinese medicine, and the ear points were determined by detecting the Nogiers' ear acupuncture points. Both mechanical palpation techniques and digital detector were used for detecting the points.

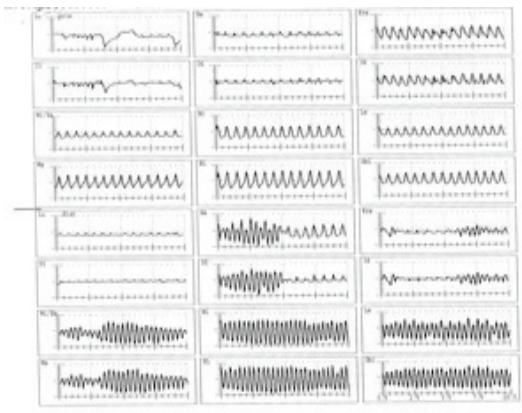

FIGURE 1: Pulse oscillography.

The investigation of pulse oscillography based on the pulse detection process was performed through a device (9).

On admission, the energy level of the patient was measured using the pulse oscillography. Blocking was observed in all three energy circles (Figure 1).

When the energy level is located above the meridians, the patient's current energy is indicated to be balanced, whereas when it is located below the 12 meridians, the patient's baseline energy is indicated to be imbalanced. The energy blockade is dramatically shown by tetany.

The following positive points in the body was identified using pulse oscillography: Shousanli (LI10) and Quchi (LI11) over the muscular origin of the lateral extensor group of the forearm; Zhouliao (LI12), one cun superolateral to LI11; Chize (LU5) in the cubital region; Taiyuan(LU9) on the radial end of the transverse crease of the wrist; Zusanlı (St 36), one finger-breadth (middle finger) from the anterior crest of the tibia; Fenglong (St 40), two finger-breadth (middle finger) from the anterior crest of the tibia; Hegu (LI4) between the first and the second metacarpal bones on

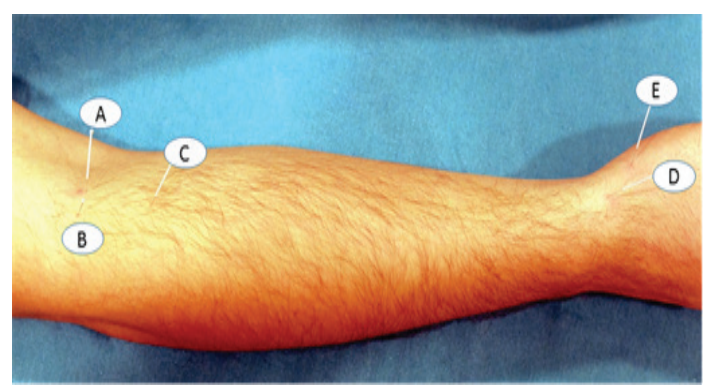

FIGURE 2: The LU5, LI11, LI10, LI5, LU9 positive points. 


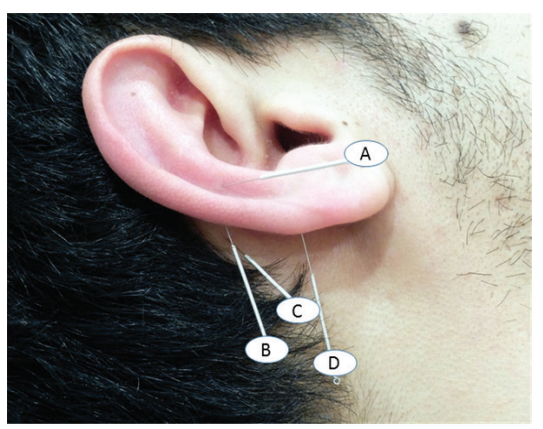

FIGURE 3: Elbow area, motor elbow point, Retro 0, and PEG 1.

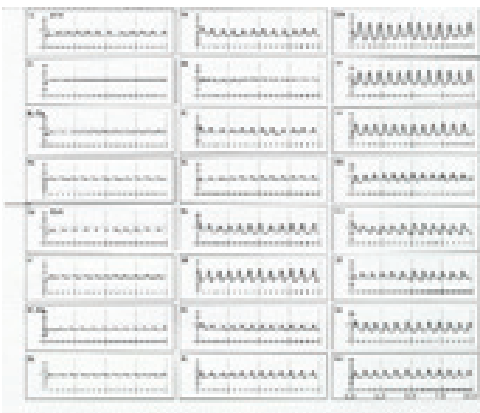

FIGURE 5: Normal pulse oscillography. the dorsal hand; Yangxi (LI5) on the radial side of the wrist; and two Ashi points were used alternately (Figure 2). Sterile 0.30 x 40 mm one-time-use acupuncture needles were used. The needles were inserted down to the musculature by manipulating the needle manually until the patient felt a warm sensation (De-qi) around the needle. The needles remained in situ for 20 minutes.

The positive points in the ear were identified using digital DT. The elbow area located on the scapha, PEG1 and voltaren points for inflammation, valium and point zero for pain and spasms in the muscles, and the motor elbow point on dorsal area were used (Figure 3). Sterile $0.20 \times 13 \mathrm{~mm}$ one-time-use acupuncture needles were applied. Needles remained in the ear for approximately 20 minutes.

Treatments were performed two to three times a week. Eight acupuncture sessions were applied within 3 weeks. The patient was examined two times at the department of physical therapy. The examination consisted of measuring the peak muscle force, pain, and functional impairment.

The 10-point VAS (visual analog scale) was used for pain measurement. The relative strength of the forearm and hand

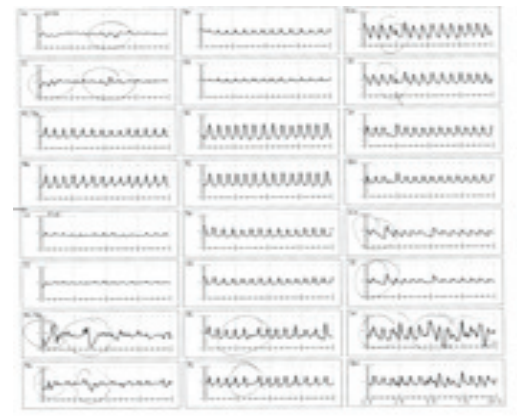

FIGURE 4: Pulse oscillography. muscles was measured using the grip strength index (10). The treatment led to significant changes in the outcome measurement, and the changes were also clinically relevant at the first follow-up. At the sixth acupuncture session, the average grip strength of the affected hand increased from 40 to 65 pounds. VAS had decreased significantly from 1000 to 400 .

Pulse oscillography indicated that the lower basal energy situation resolved blockade was observed in all three circles but irregularities were found to be more partial energy. Tetani table completely disappeared, but partial equilibrium was was on going as part (Figure 4).

At the end of the therapy, the average grip strength of the affected hand increased from 65 to 95 pounds. VAS had decreased significantly from 400 to 000 by the end of the treatment. The pulse oscillography indicated that the blocking and energy imbalance in the three circles were completely dissolved (Figure 5).

\section{DISCUSSION}

$L E$ is one of the most common diseases of the arm with repetitive microtrauma by overuse of the wrist $(1,2,3)$. Several conventional therapies are offered for tennis elbow $(4,5)$.

In the present case, the patient could not benefit from medical and physical therapies. Therefore, an acupuncture treatment was started.

Acupuncture is accepted by both clinicians and consumers of health care for treating musculoskeletal pain. Previous studies have confirmed that acupuncture has been effective in LE (11-14).

Traditional Chinese Medicine (TCM) hypothesizes that tennis elbow is the result of chronic strain damaging the normal 
circulation of Qi and blood in the local channels and collaterals. So, Qi stagnation and blood stasis cause pain and impair the functions of the elbow joint. Acupuncture is a specified TCM treatment method based on the principles of regulating yin and yang and removing Qi stagnation and blood stasis of the channels and collaterals (15). The large intestine meridian runs upward along the lateral anterior side of the forearm and reaches the lateral elbow. In the present case, the LI10, LI11, and LI12 were used to treat LE. The tender spot was also used as the Ahshi point, which is the reactionary spot of pathological change and the affected location.

Acupuncture is thought to confer an analgesic effect, and several hypotheses as to the chain of events resulting in analgesia from acupuncture have been proposed. Some authors attributed the analgesic effects to the release of b-endorphins in the lumbar spine and increased the 5-hydroxytryptophan level in the cerebrum. Other explanations include the overriding of the pain stimulus by the biochemical lines of acupuncture in the transmitting process of the central nervous system and the more traditional explanation of the freeing of a blockage of "' $\mathrm{Q} \mathrm{i}^{\prime \prime \prime}$ or energy flow by acupuncture (16-19). Acupuncture harmonizes the Qi and blood so as to restore the equilibrium of yin and yang (18). Acupuncture can be used in arthritis, pain, and rheumatoid diseases (20). All the studies suggested that acupuncture was effective in the short-term relief of the LE pain (18).

Investigating pulse oscillography based on the pulse detection process was performed through a device (9). The clinical and physical examinations have been correlated with pulse oscillography results. The pulse oscillography demonstrates the importance of diagnosis and follow-up of the treatment. The use of this device was one of the objective criteria for the treatment.

In conclusion, acupuncture is an effective therapy for $L E$, and pulse oscillography is an important device for the diagnosis and therapy.

\section{REFERENCES}

1. Shiri $R$, Viikari-Juntura $E$, Varonen $H$, Heliövaara M. Prevalence and determinations of lateral and medial epicondylitis: a population study. Am J Epidemiol 2006;164:1065-74.

2. Hamilton PG.The prevalance of humeral epicondylitis: Am Acad Fam Physicians 2007;36:464-465.

3. Orchard J, Kountouris A.The management of tennis elbow.BMJ 2011;342.
4. Buckup K. Clinical tests for the musculoskeletal system: examinations-sign-phenomena. 2nd ed. Stuttgart: NY Thieme; 2008:129-33.

5. Behrens SB, Deren ME, Matson AP, Bruce B, Green A. A Review of modern management of lateral epicondylitis. Phys Sportsmed 2012;40:34-40.

6. Jarvis WT. Quackery: a national sacndal. Clin Chem 1992; 38:1574-86

7. Smidt $N$, Assendelft W, Arola $H$, Malmivaara A, Green $S$, Buchbinder R, Windt D, Bouter L. Effectiveness of physiotherapy for lateral epicondylitis: a systemic review. Ann Med. 2003;35:5162.

8. Green S, Buchbinder R, Barnsley L, Hall S,White M,Smidt $\mathrm{N}$,Assendelft W.Acupuncture for lateral elbow pain. Cochrane Database Syst Rev 2002;1.

9. G. Silberbauer. Pulsoszillographie - die objektive Messung der Energie in den Meridianen. Ausgabe 4/2001.

10. Su X, Wu ZQ, Cao XM. [Effects of electroacupuncture of different frequencies for treatment of patients with refractory tennis elbow syndrome]. Zhongguo Zhen Jiu 2010;30:43-5. Chinese.

11. Brattberg G.Acupuncture therapy for tennis elbow.Pain 1983;16:285-8.

12. Davidson JH, Vandervoort A, Lessard L, Miller L. The effect of acupuncture versus ultrasound on pain level, grip strength and disability in individuals with lateral epicondylitis: a pilot study. Physiother Can 2001;53:195-202.

13. Grua D, Mattioda A, Quirico P, Lupi G, Allais G. L'agopuntura nel trattamento dell'epicondilite laterale: valutazione dell'efficacia e confronto con ultrasuonoterapia. [Acupuncture in the treatment of lateral epicondylitis: evaluation of the effectiveness and comparison with ultrasound therapy]. G Ital Riflessot Agopunt 1999;11:63-9.

14. Gu SP. Clinical observation on 120 cases of lateral epicondylitis treated by fire needle therapy.J Clin Acupunct Moxibust(Chin) 2003;19:12-14.

15. Induction of Hyperalgesia in Pigs through Blocking Low Hydraulic Resistance Channels and Reduction of the Resistance through Acupuncture: A Mechanism of Action of Acupuncture, Wei-BoZhang, Yi-Hui Xu, Yu-Ying Tian, Hong Li, Guang-Jun Wang, Tao Huang, and Shu-Yong Jia.Hindawi Publishing Corporation Evidence-Based Complementary and Alternative Medicine 2013:15-23

16. Viola Leonardo. A critical review of the current conservative therapies for tennis elbow (lateral epicondylitis). ACO 1998;7:53-67.

17. Zhao ZQ. Neural mechanism underlying acupuncture analgesia. Prog Neurobiol. 2008; 85:355-75. Review.

18. Trinh KV, Philips SD, Ho E, Damsma K. Acupuncture for the alleviation of lateral epicondyle pain: a systematic review. Rheumatology (Oxford) 2004 ;43:1085-90.

19. Liu N, Ren $T$, Xiang Y. Immediate analgesic effects of tendon acupuncture on soft tissue injury. Zhongguo Zhen Jiu. 2015;35:927-9.

20. Lee MS, Pittler MH, Shin BC, Kong JC, Ernst E. Bee venom acupuncture for usculoskeletal pain: a review. J Pain. 2008;9:28997. 